\title{
Synthesis of Linear and circular array antennas using gatool
}

\author{
T. Jaya Kumar ${ }^{1}$, Mrs. M. S. Anuradha ${ }^{2}$ \\ (2/2 M.Tech, ECE Dept, AUCE (A), Visakhapatnam, AP, India) ${ }^{1}$, \\ (Senior Assistant Professor, ECE Dept, AUCE (A), Visakhapatnam, AP, India) ${ }^{2}$,
}

\begin{abstract}
The main aim of this paper is to reduce the first four side lobe levels (SLLs) of the linear and circular array antennas using gatool, which is the one of the simplest and efficient tool in MATLAB ${ }^{\circledR}$. This is employed in Radars and communications in order to reduce jamming effects, EMI and heavy clustering effects. Here amplitude of the elements of the antennas is taken as variables to control. And finally the design currents are given and sum and difference pattern plots for different number of elements for linear and circular array antennas. Simulation results are presented for above with rectangular plots. Successful applications show that gatool can be used as a general tool for pattern synthesis of arbitrary arrays.
\end{abstract}

Key words: arbitrary arrays, EMI, gatool, sum and difference patterns, and simulation.

\section{Introduction:}

Low side lobe level (SLL) antennas are becoming an increasingly important component of high performance electronic systems, particularly those operating in heavy cluster and jamming environments [1-3]. The applications of GA are now common in complicated EM problems. In designing arrays with many elements (more than 20), GA is one of efficient methods which is capable of handling complex problems with many independent variables. Traditionally optimization methods are not well suited for optimizing large arrays, including higher gain and a single element, electronic beam steering and control over the antenna pattern [4]. In many conventional design techniques, the amplitude and phase excitation are obtained for elements with equal separations and the far field pattern of the array is controlled by the amplitudes and phases of the excitation of currents. In some other methods the position of the elements is controlled with an assumed current distribution. In optimization, using GA there is no limitation on selection of variables. Therefore the amplitudes of the excitations can be used as independent variables in the optimization process. The purpose of optimization of antenna array patterns is reducing the half power bandwidth, SLL or null position control of the pattern. In this paper, the reduction of first four SLL and all SLLs by controlling the excitation of currents for Linear and Circular array antennas are performed.

\section{SLL Optimization}

Linear array antennas [5]: A single antenna has a limited radiation pattern. But with the use of several antennas working together (array), it is possible to improve the radiation according to some specifications. In general the characteristics of the array are controlled by the proper choice of the element (dipole, horn, patch etc.), the geometry of the array and the excitation (amplitude and phase) of each element that satisfy a set of specifications on the beam pattern.

An array of identical elements all of identical magnitude and each with a progressive phase are referred to as a uniform array. Let us assume that all the elements have identical amplitudes but each succeeding element has a $\square$ progressive phase lead current excitation relative to the previous one, shown below.

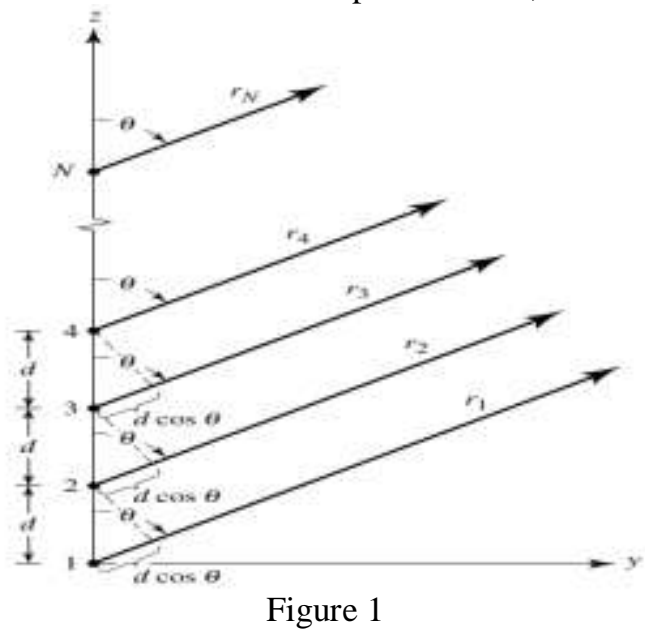


The normalized array factor is given by

$$
(\mathrm{AF})=\sum_{\mathrm{n}=1}^{\mathrm{M}} \mathrm{a}_{\mathrm{n}} \cos \left[\frac{(2 \mathrm{n}-1)}{2} \mathrm{kd} \cos \theta\right]
$$

Where $a_{n}$ is the excitation coefficients of the array elements, $k$ is the wave number, $k=2 \pi / \lambda, \theta$ is the axis angle measured form the axis of the array. The fitness function associated with this array is the maximum SLL of its associated far field pattern to be minimized

Circular array antennas: Circular arrays offer several advantages over linear arrays in direction finding applications. They provide 360' azimuthally coverage, and can provide information on elevation angles of sources. In contrast, linear arrays can provide only 180' azimuthally coverage, and cannot provide information on source elevation angles. Also, directional patterns synthesized using circular arrays can be rotated through 360 ' of azimuth without significant change of shape. Our use of the circular array geometry stems from these advantages [6-7]

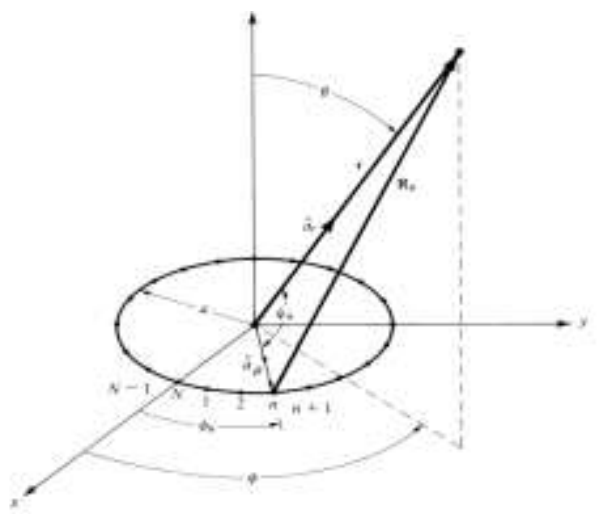

Figure 2

Array factor: let us assume that $\mathrm{N}$ isotropic elements are equally spaced on the $\mathrm{x}-\mathrm{y}$ plane along a circular ring of the radius a. The normalized field of the array can be written as

$$
\begin{aligned}
& \mathrm{E}_{\mathrm{n}}(\mathrm{r}, \theta, \phi)=\sum_{\mathrm{n}=1}^{\mathrm{N}} \text { an } \frac{\mathrm{e}^{-\mathrm{jkRn}}}{\mathrm{Rn}}[\operatorname{AF}(\theta, \phi)] \\
& \text { Where } \mathrm{AF}(\theta, \phi)=\sum_{\mathrm{n}=1}^{\mathrm{N}} \mathrm{a}_{\mathrm{n}} \mathrm{e}^{\mathrm{j}[\operatorname{kasin} \theta[\mathrm{ks}(\varphi-\varphi n)+\alpha \mathrm{n}]}
\end{aligned}
$$

Above equation represents the array factor of a circular array of $\mathrm{N}$ equally spaced elements.

\section{Fitness function:}

In order to achieve the objective of reduction first four side lobe levels (SLLs) following fitness function is used

$$
\text { fitness }=20 \log 10 \frac{\max \mid\left(\left.\operatorname{AF}_{\mathrm{SLL}}(\theta, \varphi)\right|_{\mathrm{SLL}=1} ^{4}\right.}{\max \mid\left(\mathrm{AF}_{\mathrm{SLL}}(\theta, \varphi) \mid\right.}
$$

For all side lobe level reduction

$$
\text { fitness }=\max \left\lfloor 20 \log 10 \frac{\left|\operatorname{AF}_{\mathrm{SLL}}(\theta, \varphi)\right|}{\max \left|\operatorname{AF}_{\mathrm{SLL}}(\theta, \varphi)\right|}\right\rfloor \text { Where }\left|\operatorname{AF}_{\mathrm{SLL}}(\theta, \varphi)\right| \text { is the antenna array side lobe }
$$

level

\section{Genetic algorithm}

A genetic algorithm is a search technique used in computing to find exact or approximate solutions to optimization and search problems. Genetic algorithms are a particular class of evolutionary algorithms (also known as evolutionary computation) that use techniques inspired by evolutionary biology such as inheritance, mutation, selection, and crossover (also called recombination). 
Synthesis of Linear and circular array antennas using gatool

Genetic algorithm was developed by John Holland (1975) over the course of the 1960s 1nd 1970s and popularized by one of his students, David Goldberg, who was able to solve a difficult problem involving the control of gas-pipeline transmission for his dissertation [8].

Foundations for GA are well known. In the past decade GA has been used in array antennas and antenna pattern design. SLL reduction for linear and circular arrays has been showed that the reduction in the Side Lobe Level (SLL, in $\mathrm{dB}$ ) is linearly related to the logarithm of the reduction in half-power beam width. In these methods, with an assumption that the position of all the elements of the array specified, the excitation current is controlled using GA. The conventional (discrete) GA routine is the following process: To get optimal results with the GA, it was found to use (in all the examples studied) a uniform initial population that the number of chromosome was a constant in the process and ranked replacement took place in each iteration. After the natural selection takes place, the genes (parents) mate to produce offspring. Mating takes place by pairing the surviving genes. Once paired, their offspring consist of genetic material from both parents. Multi point crossover is selected for two parent genes and mutation happen in each iteration, affecting each bit on every chromosome. The mutation help the algorithms avoid a local minimum. In a chromosome mode, similar to a DNA, genes are like a chain. The GA was coded in binary and floating points. The progress of reproduction and survival selection continues until a satisfied result is obtained or preset maximum number of iteration reached. The GA package used provided Mat Lab ${ }^{\circledR}$.

\section{Genetic Algorithm Solver [9]}

We invoke optimization tool box, gatool by typing following command in the command window gatool, and it displays the GA Solver Tool box(as in fig.3). We can solve the genetic algorithm by typing the command below also

$[\mathrm{x}, \mathrm{feval}]=\mathrm{ga}(@$ fitnessfen, nvars, LB, UB)

It returns feval, the value of the fitness function fitnessfen at the solution $\mathrm{x}$. Where inputs include fitness function which is going to solve, number of variables here is currents, Lower Bound, Upper bound.

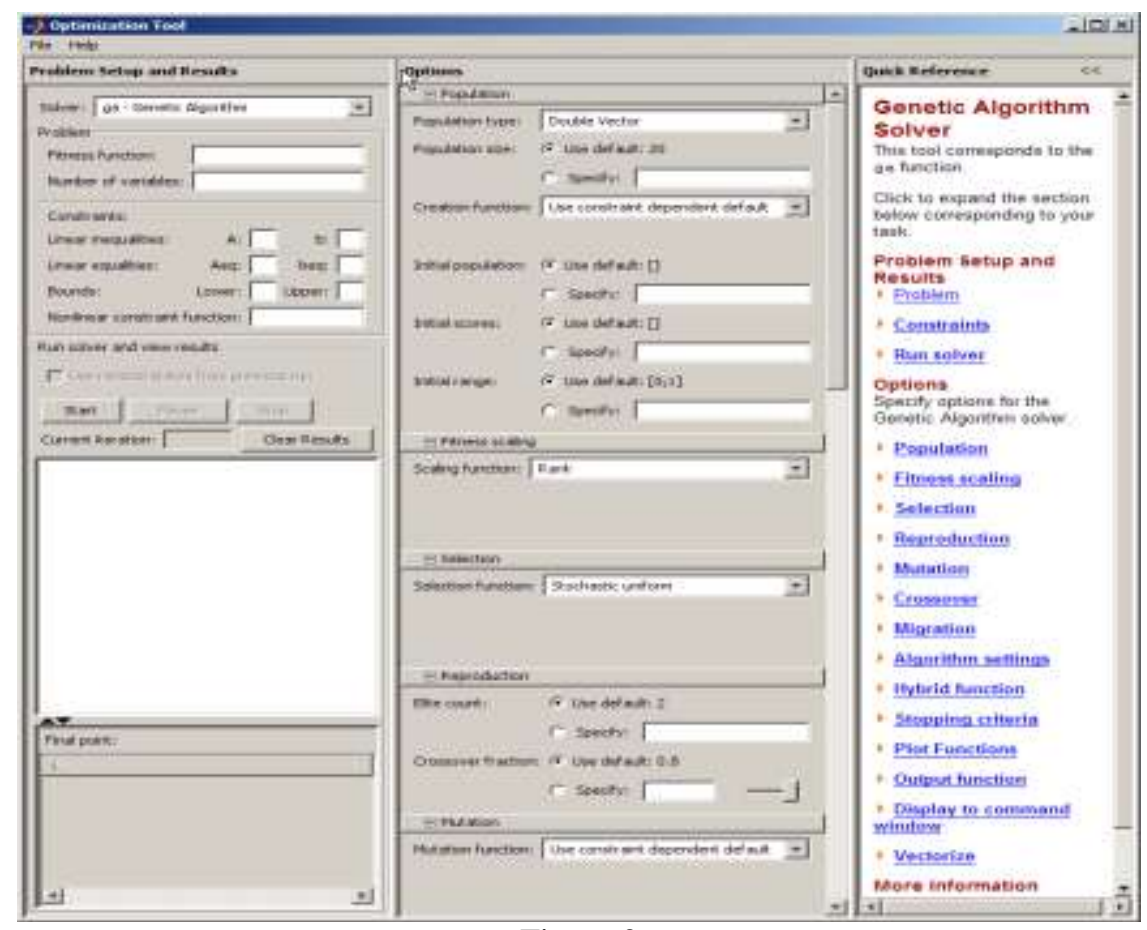

Figure 3

\section{Experimental results}

The antenna model consist of 20 and 50 elements of linear and circular array, equally spaced with $\mathrm{d}=0.5 \lambda$ for linear array and equal circular array. The amplitude of the voltage level is the antenna weight. Only the voltage applied to the element is changed to find the amplitude distribution, while the array geometry and elements remain constant.

Optimization toolbox with ga-Genetic Algorithm solver in MATLAB ${ }^{\circledR}$ has been used in experiments to find the amplitude excitations to achieve the minimum side lobe levels.

By invoking the gatool population size $=20$, Selection function=Roulette, Reproduction (Elite count) $=1$, Mutation function=Adaptive feasible and Cross over function=Single point are set by default. We can vary these 
as per our requirement, while running the fitness function also. Number of variables is the amplitude weights here $\mathrm{N}=20$ and $\mathrm{N}=50$ here taken. Here author used the default values and obtained the results. By individually solving for each fitness function all are plotted in other program. We also can do it by using Multi-objective function. There are numerous options are there for obtaining the required solutions, by varying then in gatool, can be observed in gatool.

Case 1: In the first case for linear array $\mathrm{N}=20$ and $\mathrm{N}=50$ the reduction of first four side lobe levels (SLLs) and reduction of all SLLs are Sum patterns plotted. Shown in Fig 4

Case 2: Here for $\mathrm{N}=20$ and $\mathrm{N}=50$ for linear array antenna, the reduction of first four side lobe levels and reduction of all SLLs of Difference patterns are plotted. Shown in Fig 5

Case 3: Here for Circular array $\mathrm{N}=20$ and $\mathrm{N}=50$, the reduction of first four side lobe levels and all SLLs are plotted.

Design currents for $\mathrm{N}=20$ for linear Array Antenna is given below. For reduction first 4 SLLs
$\mathrm{In}=[0.4898$
0.0599
0.1090
$0.3107 \quad 0.2218$
0.1225
0.3779
0.4267
$0.9680 \quad 0.0785$
0.9711

$\begin{array}{lll}0.1199 & 0.9036 & 0.3632 \\ \text { For reduction of all SLLs }\end{array}$
$\mathrm{In}=[0.1210$
$0.1944 \quad 0.2380$
$0.1847 \quad 0.3284$
0.48420 .6407
$0.5283 \quad 0.5225$
$0.6139 \quad 0.8139$
0.66070 .9422

\section{Plots: For Linear Antennas Arrays Sum patterns}
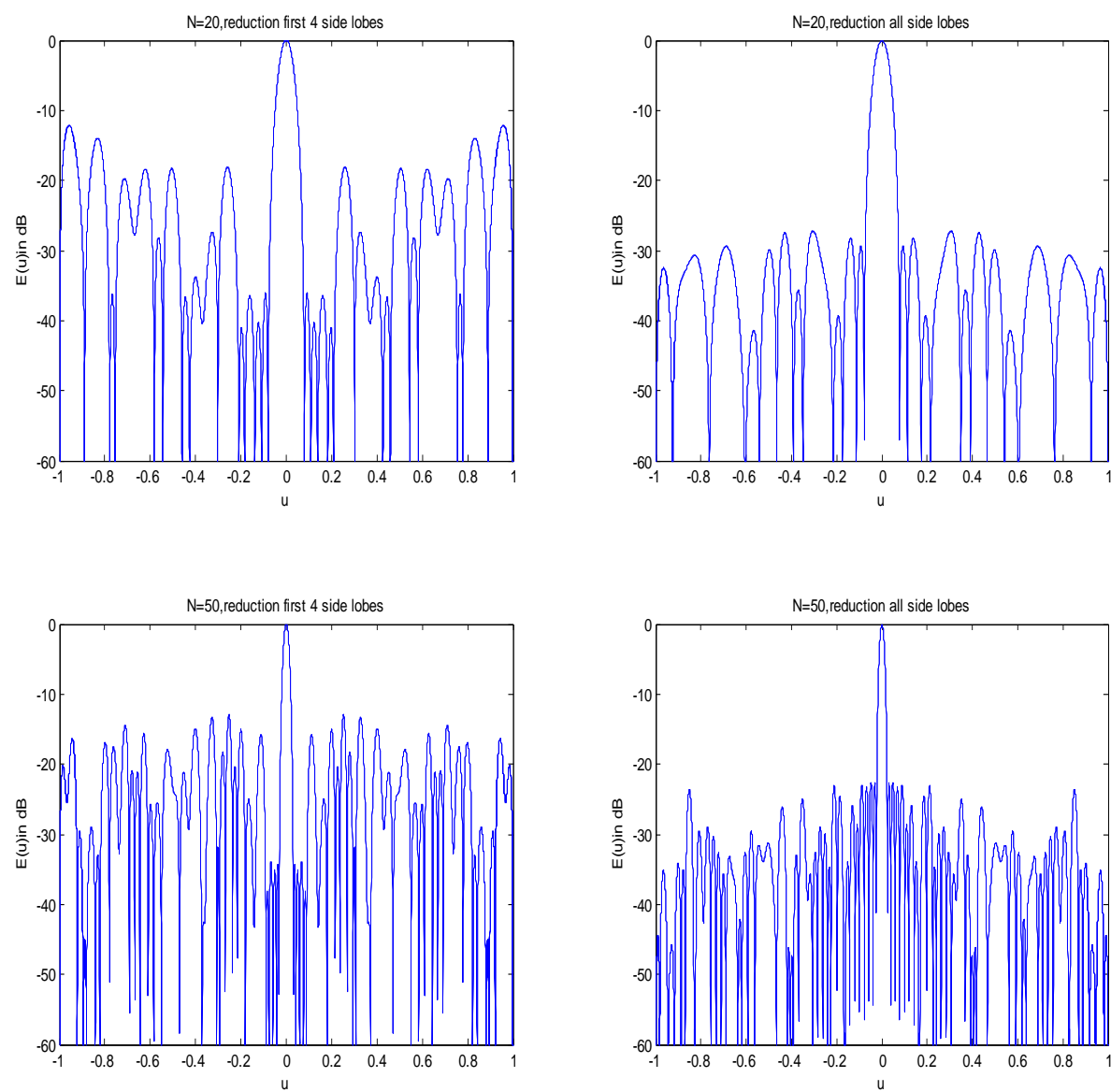

Figure 4 


\section{For Linear Antennas Arrays Difference patterns}
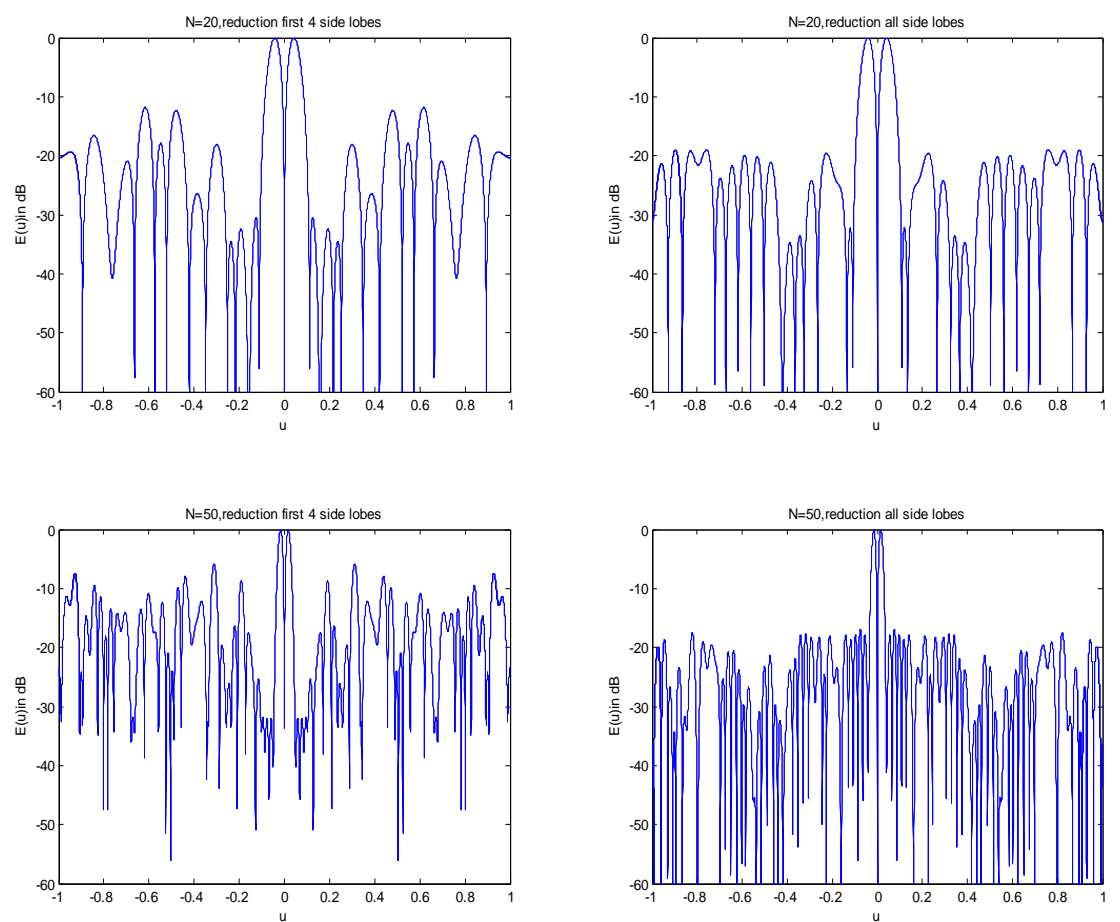

Figure 5

\section{For Circular Antenna Arrays}
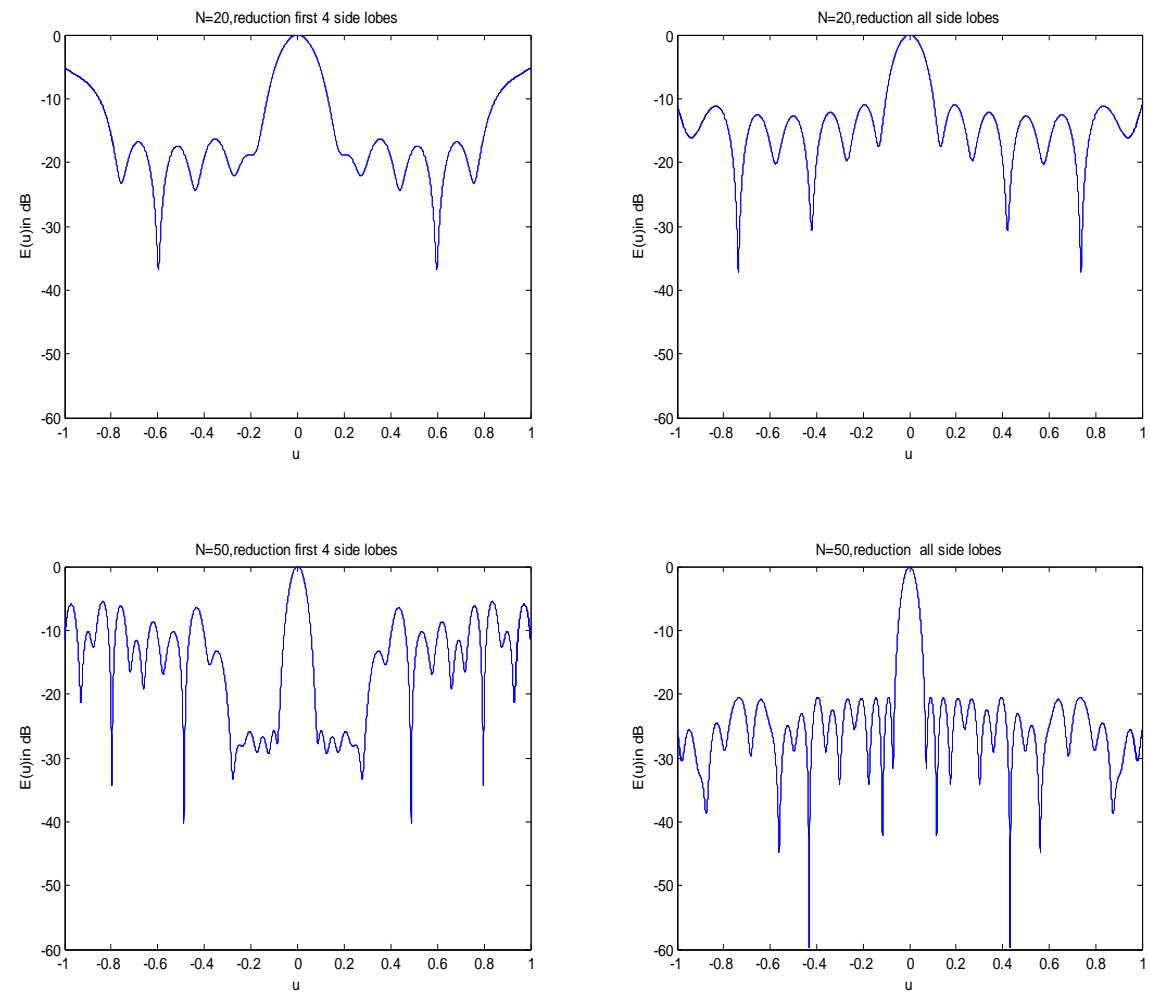

Figure 6 
Table for time taken using ga in different aspects for reduction of all side lobes using floating point is shown below.

Table 1

\begin{tabular}{|l|l|l|}
\hline & For $\mathrm{N}=20$ & For $\mathrm{N}=50$ \\
\hline Using gatool & 55.80 secs & 110.09 secs \\
\hline $\begin{array}{l}\text { Using ga in command } \\
\text { window }\end{array}$ & $\begin{array}{l}\text { Approx same time as } \\
\text { above }\end{array}$ & $\begin{array}{l}\text { Approx same time as } \\
\text { above }\end{array}$ \\
\hline Using program & 122.777341 secs & 261.246490 secs \\
\hline
\end{tabular}

\section{Conclusions:}

By employing the above, we can reduce the jamming and EMI problems effectively. This paper has shown how to apply a gatool to find low side lobe levels (SLLs) that take into account antenna currents, gatool has been found to be well suited to apply to several problems of array antenna pattern synthesis. To improve the radiation pattern and the AF, a combination of array variables (amplitudes) was controlled in such a way with an increase in the dimension of the problem, for optimizing of the side lobe level antenna using GA. Two problems for linear and circular arrays with dimensions 20 and 50 were analyzed using gatool and the results were plotted. Table for time taken using ga in different aspects for reduction of all side lobes using floating point is shows that using the gatool is the fastest and best method to find solutions. By employing the gatool we can save time, complexity, space and manual errors. The same concept can be applied for planar and concentric circular antenna array also.

\section{References:}

[1]. "Side Lobe Level Optimization in Phased Array Antennas Using Genetic Algorithm", by F.Soltankarimi, J.Nourinia, and Ch.Bhobadi, ISSSTA2004, Sydney, Australia, 30 Aug.-2 Sep.2004.

[2]. Harrington, R. F., "Side lobe Reduction by Non-uniform Element Spacing," IRE Trans.,mVol. AP-9, March 1961, pp. 187-192.5 PANDURO, M.Mendez, A. Dominguez, R. ROMERO, G. Design of no uniform circular antenna arrays for side lobe reduction using the method of genetic algorithms, Int. J.Electron. Commun. (AEU) 60 (2006), 713- 717.

[3]. Sandler, S. S., "Some Equivalences Between Equally and Unequally Spaced Elements,'” IRE Trans. Vol. AP-8, September 1960, pp. 496-500

[4]. Cyber Journals: Multidisciplinary Journals in Science and Technology, May Edition, 2011"

[5]. Investigation on the Performance of Linear Antenna Array synthesis using Genetic Algorithm" T.S.JEYALI LASEETHA1 Dr. (Mrs.) R.SUKANESH2

[6]. H.D.Griffiths "The use of circular arrays for direction finding applications, IEEE transactions.

[7]. Ali Abdulhadi Noaman , Dr Abdul Kareem, S.Abdallah, Dr. Ramzy S.Ali, "Optimal Side lobes Reduction and Synthesis of Circular Array Antennas Using Hybrid Adaptive Genetic Algorithms.

\section{Books}

[8]. Antenna theory analysis and Design by Constantine A.Balanis Second Edition Wiley India Edition, 2008

[9]. Practical Genetic Algorithms by Haupt R.L., Haupt Sue Ellen, Second Edition Wiley Interscience Publications, 2005.

[10]. Genetic Algorithms in Search, Optimization and Machine Learning by David E. Goldberb, Second Edition, Wiley Interscience Publication,2005

[11]. Genetic Algorithm and Direct Search Tool box, Mat Lab® manual 